\title{
Energy Saving by Means of Fuzzy Systems
}

\author{
José R. Villar ${ }^{1}$, Enrique de la Cal ${ }^{1}$, and Javier Sedano ${ }^{2, \star}$ \\ ${ }^{1}$ Computer Science Department, University of Oviedo, Gijón, Spain \\ villar@uniovi.es, delacal@uniovi.es \\ ${ }^{2}$ Electromechanic Engineering Department, University of Burgos, Burgos, Spain \\ jsedano@ubu.es
}

\begin{abstract}
It is well known that global sustainability must begin with human actions. A reduction of the consumed energy in the heating systems is one of such possible actions. The higher the society prosperity the higher the required houses comfort, and the higher amount of energy. In Spain it is especially important as the construction rate is almost the half of that in Europe. To save energy is urgent, which means that the energy losses must be reduced.

In this paper, a multi agent system solution for the reduction of the energy consumption in heating systems of houses is presented. A control central unit $(\mathrm{CCU})$ responsible of minimising the energy consumption interacts with the heaters. The CCU includes a Fuzzy Model (FM) and a Fuzzy controller (FC) and makes use of the concept of energy balance to distribute the energy between the heaters.

Results show the proposed system as a very promising solution for energy saving and comfort tracking in houses. This solution is the preliminary study to be included in a heating system product of a local company.
\end{abstract}

\section{Introduction}

The building rate is increasing all over the world, and this determines the resources consumption in the planet. The emerging economies are the high resources consumers, so they produce a huge impact in environment pollution [10]. For instance, the $28.4 \%$ of buildings in Europe were constructed in Spain during 2005 [1. According to this, from 1990-2007 the trend of emissions in Spain will surpass in 20 percentual points the spanish limit in the Kyoto Agreement [2].

A direct consequence are the policies to impulse the reduction of energy consumption. In particular, the energy consumption reduction in the construction of buildings is not defined yet 4917]. In buildings, heating systems represent the main energy consumption source. The thermal comfort in houses is also increasing, so more energy will be spent in heating systems.

Energy saving systems must manage a lot of variables in order to maximize the comfort while the energy needs are minimized 6651012]. Therefore, simulation

\footnotetext{
* This work was funded by Spanish M. of Education, under the grant TIN2005-08386C05.
} 
tools are used for estimating the energy consumption and the comfort measures in architectural projects. Building design simulation tools can be divided in equation based models [710911, and third part developed tools based, 4[17]. The equation based models are suitable when building materials or new construction procedures are analyzed. Simulation tools based on concentrated parameters models are more suitable when modeling the energy consumption. Some common tools based on concentrated parameters are HTB2 [18] and HOT2000 [19.

In apart, a new catalogue of dry electrical heaters will be marted by a local company in late 2007. In this work, a new Fuzzy Energy Saving Domotic System will be designed as a complement for such a product, with the aim of saving energy while keeping the comfort in the house.

This proposal includes a distributed architecture based on several distributed agents (the heaters) and a Central Control Unit (CCU). Both the CCU and the heaters will communicate using Zigbee technology. An energy saving and distributor algorithm, which makes use of a fuzzy model and a fuzzy controller, is implemented in the CCU. The energy requirements of the comfort specifications in each room is determined by means of the fuzzy model, while the fuzzy controller is used to calculate the heating power to be applied in each heater. Finally, this Energy Distribution Algorithm (EDA) determines how to share the contracted energy between the heaters in the house to achieve the desired comfort temperature level.

This work is ordered as follows. In the next section the problem is described. In Sect. 3 the Fuzzy Energy Saving Domotic System is analyzed and explained. The experiments run and the results obtained can be seen in Sect. 4 Finally, the conclusions and proposed future works are given.

\section{The Problem Definition}

The main goal is the design of a system for saving electrical energy for keeping the comfort level of the house considering the remaining electrical installations (but the heating installation) and the contracted electrical power. The comfort level in a house is defined as the ambient variables that the user fixes for each room: each room temperature, humidity level, etc. Houses can be constructed using different kinds of materials, different topologies, from different geographical zone, etc. Thus, here we define the standard house in Spain in Fig. 1 .

Each room in the house will have at least one heater, with a given power rate. The number of heaters and the power installed in each space is defined by the spanish comfort regulations [3]. It is assumed that each heater will collaborate with the $\mathrm{CCU}$ in order to reduce the consumed energy while keeping the comfort in the house. The comfort specifications for each room must be given, establishing the temperature set point for every hour and day of the week. Moreover, the electrical energy contract for each house limits the amount of energy that could drain in a house. Currently, the most usual rate in Spanish houses is $4.4 \mathrm{KWh}$. Besides, the sum of the electrical power of each heater plus the power of the rest of the electrical devices in the house must not exceed the former limit. Finally, 

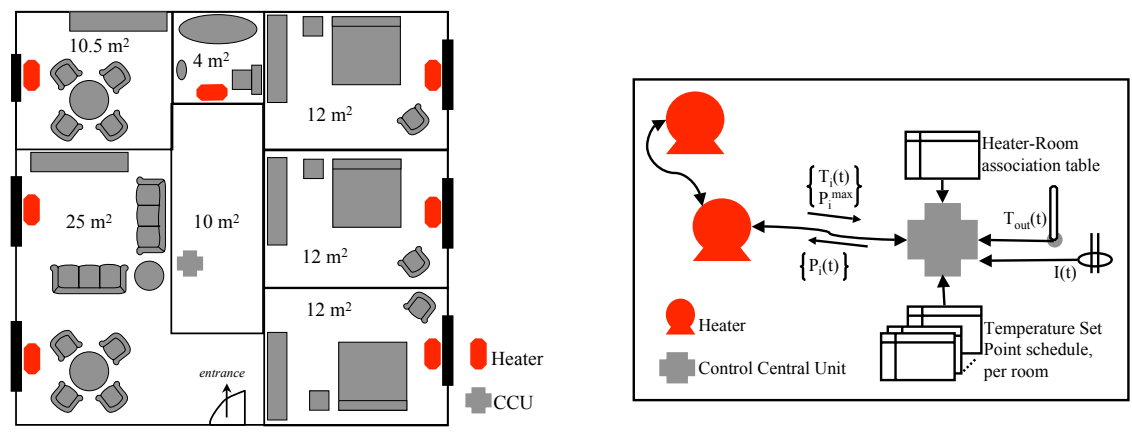

Fig. 1. The standard house designed and the architecture schema. The heaters are the gray boxes close to the windows. The CCU is in the corridor. The multiagent system schema. The CCU receives all of the information about the comfort variables from each heater. The CCU also computes and distributes the instantaneous maximum power for each heater.

the whole saving energy system must be economically viable so that consumers can afford it. Also, the system must be robust so failures in the CCU do not collapse the system.

As stated above, the model is to be included in a heating system product of a local company. The possible power rates for the heaters are fixed by fabric catalogue, and they could be 500, 1000, 1200 and 1500 Watts.

\section{The Fuzzy Model for Energy Saving and Comfort}

In this work, a multiagent system is proposed as a solution for the problem defined in the previous section. In few words, the heaters send to the CCU the temperature from the room, and its power rate. The CCU measures the instantaneous consumed current and the outdoor temperature. The CCU also stores the set point temperature profiles for all of the rooms in the house, and the association between rooms and heaters. Finally, the CCU computes and distributes the instantaneous maximum power for each heater. This power distribution is calculated to optimize the energy consumption in the house. In the right of Fig. 1 it is shown a schema of the system.

The power distribution between the heaters is carried out by means of an energy distribution algorithm, which makes use of a fuzzy model and a fuzzy controller to fit the maximum power for each radiator. A block diagram of the whole process is shown in Fig. 2. There are two stages in the solution: the design stage and the run stage. In the design stage, a fuzzy model (FM) and a fuzzy controller (FC) are generated. The FM has to estimate the power requirements of a generic room, and the fuzzy controller (FC) has to fit the power of a heater. To generate the learning datasets for the FM the simulation software tool HTB2 [18] has been used. The FC was designed ad hoc. 


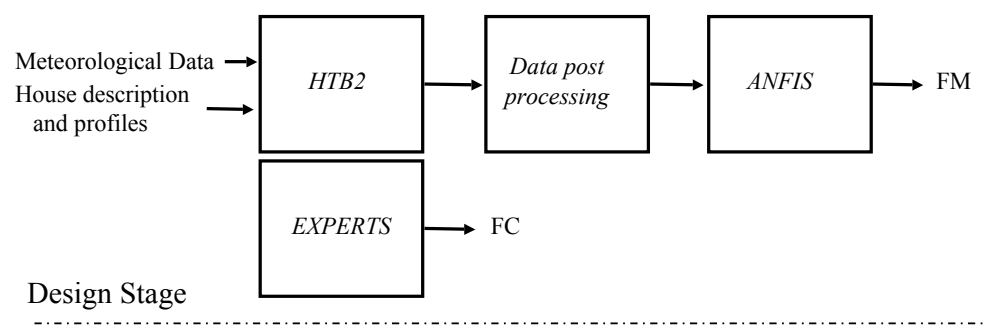

Run Stage

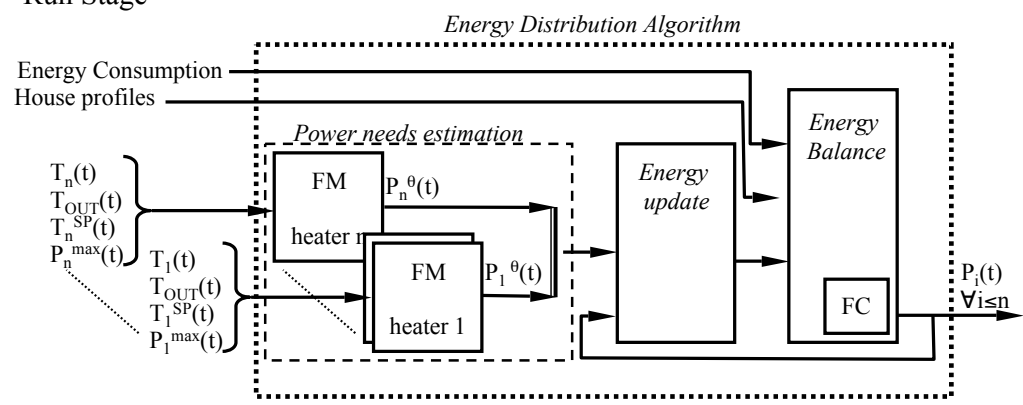

Fig. 2. The prototype black boxes diagram. In the design stage, the fuzzy model (FM) and the fuzzy controller (FC) are designed. In the run stage, the instantaneous maximum power for each heater is computed and distributed.

The instantaneous maximum power for each heater has to be determined in the run stage. The CCU receives the power rate and the temperature of the room from all of the heaters, and estimates the power requirements of each one by means of the FM. Then, the estimated energy required for heating the room and the energy used in heating the room are calculated. Finally, a balance of energy is used to infer the instantaneous heating power for each heater. The FC is used to calculate the heating power required for each heater, knowing already the room temperature error and the energy error.

The whole solution is detailed as follows. The next subsection deals with the analysis of the solution, while in Sect. 3.2 the multiagent system is roughly described. The FM and the energy distribution algorithm are detailed in Sect. 3.3 and Sect. 3.4.

\subsection{The Solution Analysis}

Before providing a solution for the problem, some definitions must be explained. For example, the actual definition of comfort, or the physical variables that must be measured, as well as the system architecture, or the degree of parameterization of the prototype.

Comfort is defined as the state of physical ease and absence of pain and constraint. In the problem that concerns us, comfort means the conditions of the environment of a house which helps the inhabitants to relax. There are several 
methods to measure the level of comfort. For example, measuring the temperature and the humidity of a room could be used to infer the level of comfort. When talking about heating systems, the constructing regulations establish the use of the temperature in a room to measure the level of comfort. The temperature is the only data used to measure the comfort. Further studies should be carried out including other date, as the humidity, for measuring the comfort level. In apart, in each heated room there is at least one heater. As each heater includes a temperature sensor, the temperature measured in each heater will be used as the room temperature. Each heater also includes a microcontroller, and a human interface.

Besides, the outdoor temperature and the instantaneous power consumption have to be measured. The outdoor temperature should to be measure in order to reduce the energy waste as much as possible, while the power consumption should be known so the energy supplier contract limit is not exceeded.

Some hints about costs are to be considered when deciding the system architecture. First, the cost for installing the connections must be kept as low possible, not only of materials but also in manpower. Second, the cost of configuring the system should be also low: the installer must configure in a limited period of time. The configuration must be simple, with a reduced set of parameters.

The electric heaters to be installed are autonomous, that is, they are the same heaters that could be installed in a house without the energy saving system. Each heater includes a microcontroller, a temperature sensor and a human machine interface for setting it up. As stated before, a CCU would be installed to control the whole saving system. But also, the CCU would be the common interface with the system, storing the set point temperature schedule, the configuration of the house, etc.

A wireless network is used with the aim of reducing the installing costs. If the heaters are extended with such capability, then they can send to the CCU the room temperature data, and their power rate data too. Also, the outdoor temperature sensor and the instantaneous power consumption sensor could be wireless, but now both are considered wired.

The configuration costs in a wireless network of heaters are reduced to the following parameters: a network address is given to each heater, provided that it is unique in the neighbourhood of the systems; then the heater can send and receive data from the $\mathrm{CCU}$.

There are some more parameters to set up in the CCU. First, assign a unique network address to the CCU. Also, name the number of rooms and their type kitchen, living room, etc.-, and the association table between rooms and heaters. The default set point temperature schedule -in following, temperature profilecan be given by software, and the final user can easily customize. A total of 27 parameters should be given for a house as that described in Sect. 2, 8 network addresses, with 6 rooms, 6 rooms types, and 7 associations room-heaters.

The Zigbee model [15] of wireless network has been chosen as all the nodes in the network are microcontrollers, and Zigbee is a low cost microcontroller network. In this network each heater acts as a node, but also could act as a repeater 
when needed, in order to get all the nodes reaching the CCU. This behaviour is dynamically determined in the network, and could not be parametrized.

\subsection{The Multi Agent System}

As stated before, in a Zigbee network each heater decides when to behave as a repeater. Moreover, a heater will be governed by the CCU when the network is up. But when the network is down, or when it detects that the CCU is out of service, the heater must act as a normal stand alone heater until the system recovers.

This is the reason why a mutiagent system paradigm is adopted in a similar way as proposed in [7. In Fig. 17right, it is shown the schema of the multiagent system, where the data flows between heaters and CCU. A heater could act as a stand alone heater, as in the network heater with or without repeater behaviour. The goal is that the house reaches the predefined comfort level.

\subsection{The Estimation of the Power Requirement in a Room}

The power requirements of a room is the instantaneous electric power needed to heat up the room, so it could reach the comfort level. But it is not possible to have a model for each different room. In the hypothetical case that it could be possible, there were too many parameters to be configured in the CCU that would made the installation unfeasible. That is the reason why the behaviour of the heating dynamics in a generic room is modeled. The estimation of the power requirements of a generic room has to deal with uncertain dynamics.

There are several reasons that induce the uncertainty. First, the geometric dimensions of a room are represented by the power rating of the heater or heaters installed in because this is the only known data from the room. Second, heating up a room depends on the weather, it is not the same to heat up a room in a cold winter than in a warm autumn. Finally, the occupancy profile, or the small power profile in each room introduces vagueness in the behaviour of a heating system. As a conclusion, the estimation of the power requirements of a generic room needs a model suitable to manage such vagueness.

Fuzzy logic is a well known technique for managing uncertainty [16. By means of a fuzzy model, the power requirements of a generic room estimation is to be accomplished. The learning of the fuzzy model is carried out in the design stage. The ANFIS model [13] has been chosen to model the power requirements of a generic room, which has been proved suitable when the dataset includes data from all kind of events need to be learned, and when it is to be used in short-mid term 814. The generation of the ANFIS model is shown in a block diagram in the Fig. 2. The HTB2, postprocessing and training steps are described in the following subsections.

Creating the Dataset from Realistic Simulations. As stated in [814], to successfully train an ANFIS model it is needed a dataset sufficiently large to include all of the situations and events be modeled. At the moment this paper 
was written, the production of the heaters in the fabric is just initiating, so that it was not possible to have a physical installation as a test bed. The dataset must be generated from simulations. The HTB2 simulation software [18, in following HTB2, is a well known tool suitable to analyze the dynamics of heating systems with concentrated parameter problems as the one that concerns us 4]17.

The HTB2 is a totally parametrized simulation tool. The main output of a HTB2 simulation is the heating power requirements for each heater and room in the modeled house, but also the temperature in each room, both logged at each time interval. The materials in the construction, the volumetric measures of each room, the neighbourhood of the rooms, the orientation and geographical earth zone, the solar radiation profile, the environment data, the heating subsystems, the occupancy profile, the temperature profile for each heating subsystem, the small power devices and the light $\mathrm{ON}$ profiles can be fixed for each room and space. Different sample periods and the length of the simulations could be fixed too.

For generating the dataset, all of the topologies of houses and geographical zones to be covered must be defined, which are market decisions. Then, each topology and geographical zone must be defined for the HTB2, and a set of simulations for each season must be carried out using realistic profiles of occupancy, set point temperature, small power devices consume, etc.

The Postprocessing Stage. The HTB2 software generates a huge dataset. Each line of the HTB2 output dataset includes data from all of the rooms at certain time interval. This dataset must be postprocessed, so each of the examples in it contains relevant data from one room only. First, a grouping step is run, so each line in the dataset contains data from only one room. Then the grouped dataset is resampled, so only relevant examples are taken into account. Relevant examples are those that include information of dynamics. For example, when a room set point temperature is 0 there is no need to be modeled as it does not have information of the dynamics.

The outcome of the postprocessing stage is the dataset for training and testing purposes, each line in such dataset contains values of the following variables for a certain room: the temperature in the room $\mathrm{T}_{i}$, the room set point temperature profile $\mathrm{T}^{S P}$, the heating power installed in the room $\mathrm{P}_{i}^{\max }$, the power requirements for the room heater $\hat{P}_{i}$, the outside temperature $\mathrm{T}_{\text {out }}$, the occupancy rate of the room $\mathrm{O}_{i}$, the light power consume of the room $\mathrm{L}_{i}$, and the small power devices consume of the room $\mathrm{S}_{i}$. The $\mathrm{T}_{i}$ is one of the outputs of the HTB2 simulations. The values of $\mathrm{T}^{S P}, \mathrm{~T}_{\text {out }}, \mathrm{O}_{i}, \mathrm{~L}_{i}$, and $\mathrm{S}_{i}$ are the same used in the HTB2 simulation. The $\mathrm{P}_{i}^{\max }$ represents all of the knowledge about a specific room, but also the maximum amount of power to be employed. The $\hat{P}_{i}$ and the $\mathrm{T}_{i}$ are the mayor outcome of the HTB2 simulations.

The Learning Stage. The learning phase of the ANFIS FM has been developed in Matlab [20]. The postprocessing output dataset will be used in training and validation, it is stratified in a $10-\mathrm{k}$ fold cross validation schema, and the training and testing of 10 ANFIS models is carried out, provided that the dataset is large enough to contain sufficient relevant information. 
As a result, 10 ANFIS models are obtained, so statistical analysis could be done. The best suite model will be chosen. The ANFIS model will estimate the power requirements of a generic room for a given environment condition. The input variables of the FM are the instantaneous inroom temperature given from a heater, the outdoor temperature, the heater power rate and the room set point temperature as stated in the comfort profile. The output of the model is the estimation of the power requirements for the room.

\subsection{The Energy Distribution Algorithm}

In Spain, the heating power in a house easily surpasses the $7 \mathrm{KW}$, despite the most common electric power rate contract in Spain is $4.4 \mathrm{KW}$ of instantaneous consume per hour. Moreover, there are always some small power devices drawing energy: the fridge, computers, etc., so that the real instantaneous available power is lower than the contract rate. At any moment a another small power device can be in use, as example, a microwave oven.

As a conclusion, the power requirements for each room should be estimated, but also the available power should be determined, in order to distribute along the active heaters and not surpass the contracted power limit. An active heater is a heater for which its associate room has a higher temperature set point than a certain threshold. Obviously, the power distribution will be efficient if the pre defined house comfort level is reached with the minimum electric energy waste.

The solution makes use of the concept of energy balance. Energy is the capacity of the heating system to keep the comfort level. The heater required power is the power that the heater must spent to keep the comfort in the room. The heater required energy is its required power by time unit. A distribution algorithm is used, so each heater is given with a fraction of the required energy that it is allowed to spent. The heating energy is the fraction of the required power that a heater is allowed to spent (heating power) by time unit. If it is desired that the room reaches the comfort level, then the heating energy must equal the required energy for each room, that is, there must be a balance between both energies. The energy balance is carried out over a predefined period of time in order to eliminate the accumulative errors. A predefined window of 20 minutes has been adopted. This time window size would be also a requisite of the implementation in microcontrollers, where the memory is limited. For computing the energy balance over de time slicing window it is needed to store the instantaneous values of power requirements and heating power for each heater. Finally, this slicing window will reduce the impact of both lost and error values.

It is important to take into account the heaters thermal dynamics and capacity. A typical heater has a 6 minutes period from cold to full power state. From full power to cold state it takes typically 5 minutes. This means heaters do not heat with full power until the end of the dynamics. Also, it gets cold quickly because of its low thermal capacity. In the distribution algorithm those facts must be taken into account. The former could be solved with a certain correction factor. The latter implies that the active heaters must be always assigned with a minimum heating power to keep it hot. This threshold must be 
determined empirically. Finally, to reduce the dynamic periods, the duty cycle must be reduced, provided it is large enough to allow them to reach the permanent state. This parameter must be determined empirically also, and it has been fixed to 3 minutes.

The adopted energy distribution algorithm is shown in the box diagram in Fig. 2 and its flow chart can be seen in Fig. 3. The first time the algorithm runs the initialization of the required energy (Er), the heating energy (Eh) and the energy error $(\Delta \mathrm{E})$ are carried out. Each minute the algorithm is run. In each run, the required power $(\mathrm{Pr})$ for each heater are estimated by means of the FM. Then, all of the energy variables are updated. When the duty cycle runs out the heating power $(\mathrm{Ph})$ for each heater is calculated. It is needed to determine the current power consumption $(\mathrm{Pc})$, and to calculate the available power $(\mathrm{Pa})$ as 0.85 times the contract power limit without the $\mathrm{Pc}$. A FC is used for computing the heating power of each heater. The FC has been designed ad hoc for this problem. Finally, the $\mathrm{Pa}$ must be distributed between the active heaters attending to the $\mathrm{Ph}$ assigned to each heater, and taking into account the correction factors described before. Doing so, the $\mathrm{Ph}$ is updated by each three minutes, while the rest of variables $\mathrm{Pr}$, Er, Eh and $\Delta \mathrm{E}$ are updated by each minute.

The FC is a mandani fuzzy model with two inputs and one output. The temperature error in a room and the $\Delta \mathrm{E}$ are the inputs. The temperature error is the difference between the room temperature and the set point temperature for such room. The output of the $\mathrm{FC}$ is the percentage of heating power to be assigned to the room heater. The variables partitions and the rules have been designed intuitively, following the ideas the experts gave. The inference method is the mean of the maximums. Future work will deals with the design of the FC by means of hybrid learning techniques.

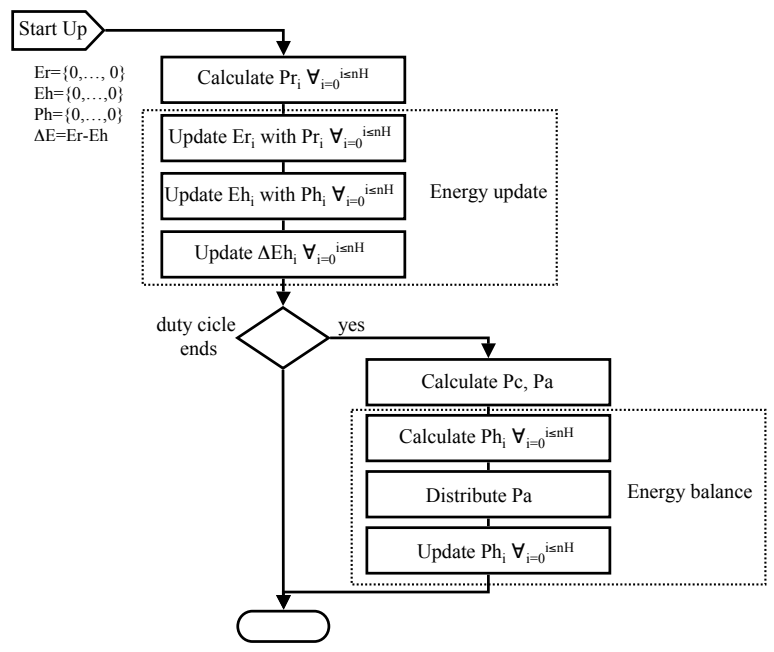

Fig. 3. The energy distributor algorithm 


\section{Experiments and Results}

In order to test the performance of the solution, a typical Spanish construction was designed and simulated making use of the house shown in Fig. 1, which is located in the north of Spain. Future works will extend the design of the FM for each area of Spain. The weather data, such as outdoor temperature, sun hours and sun radiation are taken from statistical data in the same location. The data was gathered from a cold year in winter time.

The study was designed for a mid height house, consequently, there must be simulated houses above and under the studied house and their corresponding heat interchange. The materials and the construction type are those considered standard in an actual house building in Spain, i.e., two $6 \mathrm{~mm}$ thick glasses with thermal isolation and standard windows.

A suitable profile was designed for each variable in the house that is human dependent. These variables are: the temperature set point in each room, the occupancy in the house, the small power devices schedule of use, the ventilation, and the switch on lights profile. The different profiles and timetables are according to the timetable in Spain. As an example, the temperature set point profile, and the occupancy profile for a living room are shown in Fig. 4 .

The simulation carried out by means of the HTB2 generated data about the power needs of each room in the house, with a sample period of 1 minute long. With the data from all of the rooms obtained from simulation, the postprocessing stage is carried out, and then the learning phase of the ANFIS FM. A $10 \mathrm{k}$-folds cross validation was used, and the validation and test errors are presented in Fig.5.
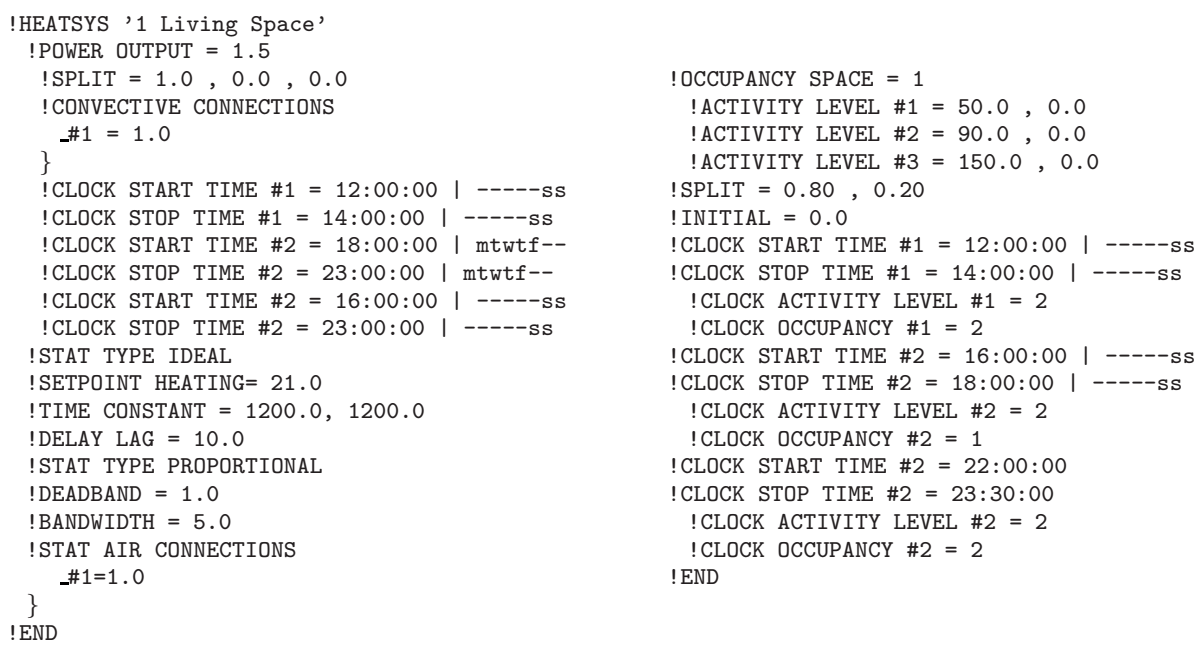

Fig. 4. HTB2 configuration file: the temperature set point -left- and the occupancy profile -rigth- for the living-room. The day of the week is specified by means of the initial letter of the day noum. A character '-' ignores the day. When no day of the week pattern is given, then the rule works for the whole week. 


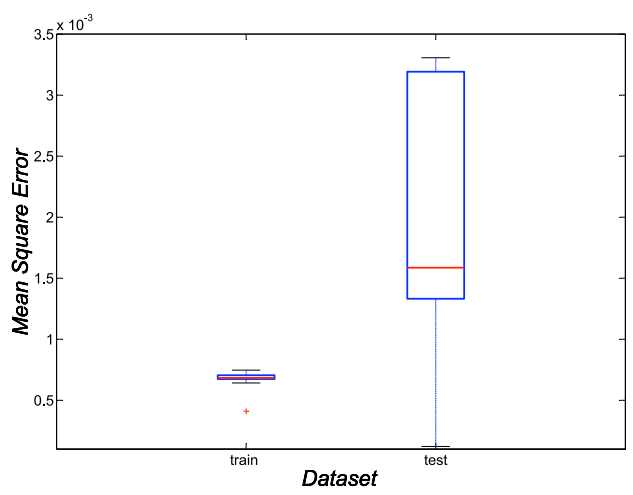

Fig. 5. The boxplot representation for the mean square error from training and testing the FM

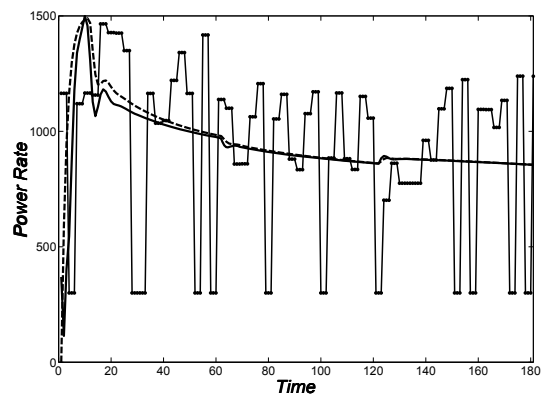

\begin{tabular}{|r|r|r|r|r|r|}
\hline Time Zone & Liv. & Kit. & Room1 & Room2 & Room3 \\
\hline \hline morning & 0 & 0.0722 & 0.0036 & 0.0196 & 0.0065 \\
\hline noon & 0 & 0.0074 & 0 & 0 & 0 \\
\hline afternoon & 0.1907 & 0.0853 & 0.0131 & 0.0221 & 0.0157 \\
\hline
\end{tabular}

Fig. 6. In the left, the control action and the energy evolution for a room and time zone: dashed line is from HTB2, continuous line is from FM, and dotted continuous line is from EDA. In the right, the Mean Square Error of the FM for different time zones and room types.

Finally, all the system has been implemented in Matlab and simulations of the behaviour of the house have been carried out. Some facts have been taken into account, i.e., the variation in a room temperature with the power rate of a heater. In order to validate the model and algorithm above detailed the error between the temperature in each room and the corresponding HTB2 simulation room temperature are to be analyzed, but also the required energy is to be compared against the heating energy. The simulations are carried out in three different day time zones.

In Fig. 6 the control action evolution for the same room an time zone is shown. In the left side, the evolution of the instantaneous power is shown. It can be seen that the FM follows the HTB2 estimation. Finally, in Table shown in the left side of Fig. 6 the mean square error for all of the rooms and time zones can be seen. 


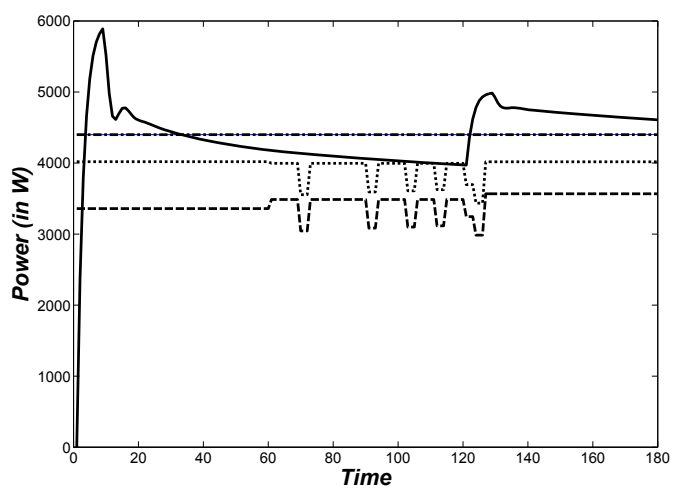

Fig. 7. The distribution and bounding action of the algorithm can be observed in the figure: bold continuous is from HTB2, dashed is from EDA, dotted is from EDA plus the small power devices profile. Straight line is the Contractual Power limit of $4400 \mathrm{~W}$.

In Fig. 7 an example of energy savings can be seen. In the shown period of 240 seconds, EDA saves $378.54 \mathrm{~kW}$ respect contracted power energy $4.4 \times 240 \mathrm{~kW}$ (a reduction of $35.85 \%$ ) and $94.082 \mathrm{~kW}$ respect HTB2 (a reduction of $9.86 \%$ ) that waste $31.847 \mathrm{~kW}$ above the $4.4 \times 240 \mathrm{~kW}$ limit (energy losts of $3.02 \%$ ).

\section{Conclusions}

Two mayor contributions can be extracted from the experimentation. First, it is possible to save energy by means of distributing the power between heaters. The consumed energy keeps lower than the contracted limits . On the other hand, the house ergonomics is preserved by means of the energy distribution when it is possible, that is, when the required energy is lower than the one available by the energy provider. Also, a percentage of available energy is preserved to perform contingencies.

Future work includes several ideas. The use of several geographical areas, different house topologies and data from different seasons must be analyzed. Also, the hybrid learning techniques must be employed to obtain a better FC. Different techniques, such as Fuzzy Genetic Systems, are expected to obtain a better result. Also, interchanging more information between the heaters and the CCU will allow a better required energy estimation and a better performance of the heating system. The humidity, as a comfort variable, must be studied as well. Finally, a real test bed must be implemented and the system must be validated.

\section{References}

1. Technical report, Euroconstruct (2007), http://www.euroconstruct.org/

2. Technical report, Ministerio de Medio Ambiente (2007), http://www.mma.es/ 
3. Boe: n. 207. Technical report, Ministerio de la Presidencia (August 29, 2007), http://www.boe.es/

4. Bojic, M., Despotovic, M., Malesevic, J., Sokovic, D.: Evaluation of the impact of internal partitions on energy conservation for residential buildings in serbia. Building and Environment (42), 1644-1653 (2007)

5. Clinch, J.P., Healy, J.D.: Valuing improvements in comfort from domestic energyefficiency retrofits using a trade-off simulation model. Energy Economics (25), 565$583(2003)$

6. Clinch, J.P., Healy, J.D.: Cost-benefit analysis of domestic energy efficiency. Energy Policy (29), 113-124 (2001)

7. Davidsson, P., Boman, M.: Distributed monitoring and control of office buildings by embedded agents. Information Sciences 171 (171), 293-307 (2005)

8. Engin, S.N., Kuvilmaz, J., Omurlu, V.E.: Fuzzy control of an anfis model representing a nonlinear liquid-level system. Neural Computing \& Applications (13), 202-210 (2004)

9. Farhanieh, B., Sattari, S.: Simulation of energy saving in iranian buildings using integrative modelling for insulation. Renewable Energy (31), 417-425 (2006)

10. Goldblatt, D.L., Hartmann, C., Dürrenberger, G.: Combining interviewing and modeling for end-user energy conservation. Energy Policy (33), 257-271 (2005)

11. Hatamipour, M.S., Mahiyar, H., Taheri, M.: Evaluation of existing cooling systems for reducing cooling power consumption. Energy and Buildings (35), 105-112 (2007)

12. Huang, C., Zou, Z., Li, M., Wang, X., Li, W., Huang, W., Yang, J., Xiao, X.: Measurements of indoor thermal environment and energy analysis in a large space building in typical seasons. Building and Environment (42), 1869-1877 (2007)

13. Jang, J.S.: Anfis: Adaptive-network-based fuzzy inference systems. IEEE Transactions on Systems, Man, and Cybernetics 23(3), 665-685 (1993)

14. Jang, J.S.: Inferring operating rules for reservoir operations using fuzzy regression and anfis. Fuzzy Sets and Systems (158), 1064-1082 (2007)

15. Kinney, P.: Zigbee technology: Wireless control that simply works. Technical report, The ZigBee Alliance (2007), http://www.zigbee.org/

16. Klir, G.J., Folger, T.A.: Fuzzy Sets, Uncertainty and Information. Prentice-Hall, Englewood Cliffs (1989)

17. Koroneos, C., Kottas, G.: Energy consumption modeling analysis and environmental impact assessment of model house in thessalonikigreece. Building and Environment (42), 122-138 (2007)

18. Lewis, P.T., Alexander, D.K.: Htb2: A flexible model for dynamic building simulation. Building and Environment (1), 7-16 (1990)

19. Natural Resources of Canada. HOT2000 User Manual (2002)

20. The Math Works. The mathworks - matlab and simulink for technical computing. Technical report, The Math Works (2007), http://www.mathworks.com/ 